\title{
Reducing energy consumption for shaping raw ceramic bricks with coordinated control of auger vacuum extruder
}

\author{
Stanislav Galitskov ${ }^{1}$, Maxim Nazarov ${ }^{1, *}$, and Konstantin Galitskov ${ }^{1}$ \\ ${ }^{1}$ Samara State Technical University, Institute of Architecture and Civil Engineering, \\ Molodogvardeyskaya str. 194, 443001 Samara, Russia
}

\begin{abstract}
This research paper focuses on the issues of enhancing technological reliability and reducing energy consumption while shaping ceramic bricks. It proves that these aims can be effectively achieved by using automatic coordinated control of actuating auger vacuum extruder systems. The paper also assesses the extent of energy consumption reductions when the extruder is equipped with an automatic control system.
\end{abstract}

\section{Problem specification}

Reduction of energy consumption while producing ceramic bricks of certain required strength is one of the main mandatory requirements to their manufacture [1]. Paper [2] proves that the quality and durability of bricks depend to a great extent on technologies of shaping raw materials. This technological process is quite energy-consuming. Generating capacity of this process can rise up to $250-250 \mathrm{~kW}$ and above. The extruder is usually controlled with the help of fairly simple algorithms. This significantly limits the ability not only to select optimal power saving modes, but also to ensure stable quality indicators of bricks formation. This paper aims to introduce effective methods and devices focused both on improving this technological process and reducing energy consumption by the installation drivers.

\section{Methodology}

The authors introduce a mathematical model of the extrusion process considering it to be a nonstationary object with distributed parameters. This model is based on the previous study of factors influencing the technology of bricks shaping as an intermediate stage of production of ceramic bricks and creating the basis for its structure and, consequently, strength [2-4]. As one of the main components of the vector of output coordinates the authors adopted shear rate $\dot{\gamma}$ of clay mixture in pressure head $[4,5]$. This is done due to the fact that value $\dot{\gamma}$ determines stability of geometric shapes, homogeneity of the internal

*Corresponding author: nazarovm86@yandex.ru 
structure and strength $R$ of fabricated bricks [2, 4]. Papers [2, 6-10] show that the required $R$ value can be achieved on conditions of coordinated control $\dot{\gamma}$ of moisture $w$ of ceramic paste and vacuum pressure of $P_{v}$ in a vacuum chamber. Let us assume that each of these process values is controlled by a separate automatic system with a minimum value of error $\Delta_{j} \quad j=1,2 \ldots m$ (where $m$ is the value of controlled technology coordinates of a vacuum extruder). To support the adopted assumption, the authors generated a multidimensional automatic control system of the extruder actuating systems (MACS EAS), such as auger electric drivers, a clay mixer and a vacuum pump. To produce ceramic bricks of an assumed strength grade $M_{i}(i=1,2 \ldots n$, where $n$ the number of bricks grades that can be produced with the extruder in question) in conditions of reducing energy consumption generated an algorithm for determining a sustainable control vector $\bar{U}_{0}$ in the sphere $0 w \dot{\gamma} P_{v}$ of control actions [7,8]. Disturbances which affect extrusion process can cause displacement of control area boundaries. This might cause either strength error $R$ or increase energy consumption. To ensure that the multi staged control system is invariant, Papers $[2,7,8]$ introduced structural synthesis of an intelligent control system for such disturbances. In this system a vector-shaping block of reference-input signal includes a data bank of extrusion process, a technological data bank of an operating unit and a correction algorithm of reference signal vector.

\section{Mathematical Model of Energy Processes}

Figure 1 presents a mathematical model of energy processes in a processing system. It helps estimate effectiveness of the proposed variant of auger vacuum extruder coordinated control. "A" here designates operators forming links between electromechanical and technological variables, durability and energy consumption: A11, A21, A31 are operators forming motors electromagnetic torques a auger $M_{d 1}$, a vacuum pump $M_{d 2}$, a clay mixer $M_{d 3}$; A12, A22, A32 are operators forming angular velocity $\omega_{a}$ of the auger, $\omega_{v p}$ a vacuum pump rotor, $\omega_{s}$ clay mixer shaft; A14, A26, A34 - are operators forming load torque on the drive motor of the auger $M_{l 1}$, a vacuum pump $M_{l 2}$, a clay mixer $M_{l 3}$; A13, A23, A24, A25, A33 - mathematical models of ceramic paste flow in a pressure head of the extruder, a vacuum pump of a suction air duct, a vacuum chamber, and a clay mixer respectively; A4 - forming unit of value $R$; A51, A52, A53 - evaluation units of specific energy consumption for operations of shaping $E_{1}^{y}$, vacuum processing $E_{2}^{y}$, ceramic paste mixing $E_{3}^{y} ; \Sigma$ - forming unit of auger vacuum extruder specific energy consumption $E_{\Sigma}^{y} ; \overline{P_{0}}-$ medium pressure ceramic paste while entering pressure head; $S_{v p}, S_{v c}$ - pumping speed at the vacuum pump output and at the vacuum chamber input; $Q_{w}$ - water flow for clay watering; $w_{0}$ - ceramic paste moisture when entering the mixer loading gate; $\mu_{1}, \psi-$ ceramic paste flow consistency index and flow behavior index at the mixer output; $Q_{m}-$ clay mixer productivity. 


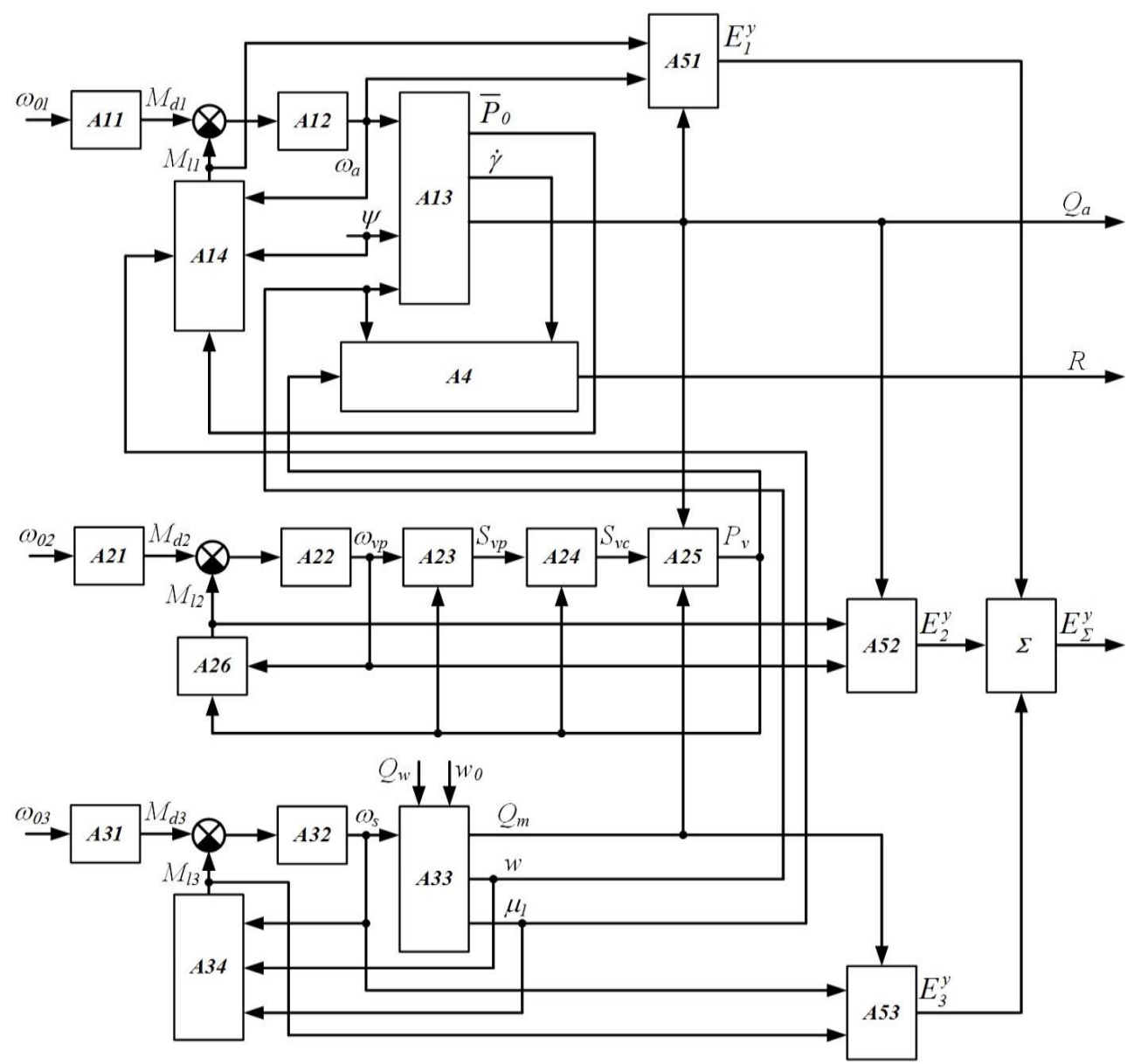

Fig. 1. Mathematical model of energy processes and its structure.

When constructing unit A51 provided a reflection of control area section $0 w \dot{\gamma} P_{v}$ [2] on the plane $0 E_{1}^{y} Q_{a}$. Here energy consumption $E_{1}^{y}$ and extruder productivity $Q_{a}$ serve as coordinate axis while producing grade $M_{i}$ bricks (as Figure 2 shows), where $P_{v}=$ const. The following functions are used in this transition:

$$
\begin{gathered}
Q_{a}=k(\psi) \cdot \dot{\gamma}, \\
E_{1}^{y}=\frac{M_{l 1} \cdot \omega_{a} \cdot i_{1}}{Q_{a}},
\end{gathered}
$$

where $k$-is the coefficient depending on the ceramic paste flow behavior index $\psi ; i_{l}-$ gear ratio of the auger drivers. ABCD area (as Figure 2 shows) is limited by the clay moisture minimum and maximum values $\left(w_{\min }, w_{\max }\right)$ and by strength $\left(R_{j}, R_{j+1}\right)$ determined by GOST 530-2012 for grade $M_{i}$ bricks. 


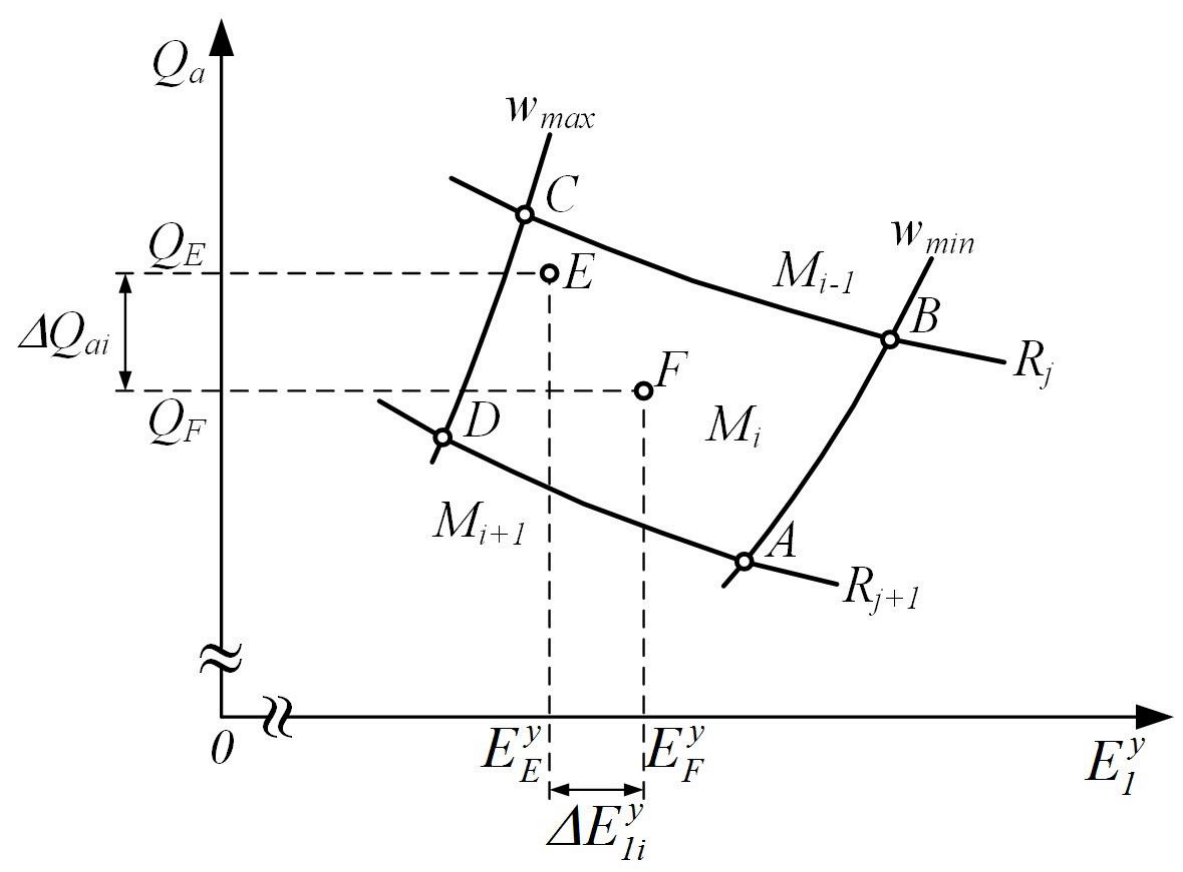

Fig. 2. The auger vacuum extruder control area while producing grade $M_{i}$ bricks.

Studying energy performance of the designed MACS EAS for two automatic control modes while producing grade $M_{i}$ bricks. The first mode is an example of traditional control involving automatic pressure leveling $\overline{P_{0}}$ of ceramic paste in the pressure head. Feed moisture $w$ in the mixer is modified according to $\overline{P_{0}}$ value, auger velocity is not modified. In the second mode MACS EAS is used. It provides coordinated control $\dot{\gamma}, w, P_{v}$ involving the use of digital observer of strength $R$.

Control mode 1 , in which the working point $\mathrm{F}$ is in the center of limited control area $\mathrm{ABCD}$, corresponds to $Q_{F}$ productivity and $E_{F}^{y}$ specific energy consumption. When we switch to mode 2 the working point moves to E-position and is located in $\varepsilon$-vicinity of Point $\mathrm{C}$ ( $\varepsilon$-vicinity is defined by $\Delta_{j}$ deviations of the extruder actuating systems) which is located on the edge of the area in question. This point corresponds to the possible attainable minimum of energy consumption $E_{E}^{y}$ and productivity maximum $Q_{E}$ while producing grade $M_{i}$ bricks. To make quantitative assessment of energy performance we introduce a relative coefficient of energy consumption reduction $K_{E i}$ and a relative coefficient of productivity increase $K_{Q i}$ :

$$
\begin{gathered}
K_{Q i}=\frac{\left|Q_{E}-Q_{F}\right|}{Q_{F}} \cdot 100 \%, \\
K_{E i}=\frac{\left|E_{E}^{y}-E_{F}^{y}\right|}{E_{F}^{y}} \cdot 100 \% .
\end{gathered}
$$

Assessment of energy performance of the designed MACS EAS as applied to the extruder with drive motors of the auger being $160 \mathrm{~kW}$, vacuum pump of $11 \mathrm{~kW}$, clay mixture of $90 \mathrm{~kW}$ while producing grades M200, M250, M300 bricks proves advantages of the 
designed control system (see Figure 3). It has also been proved that reducing energy consumption while increasing productivity of the extruder at the same time depends on grade of bricks.

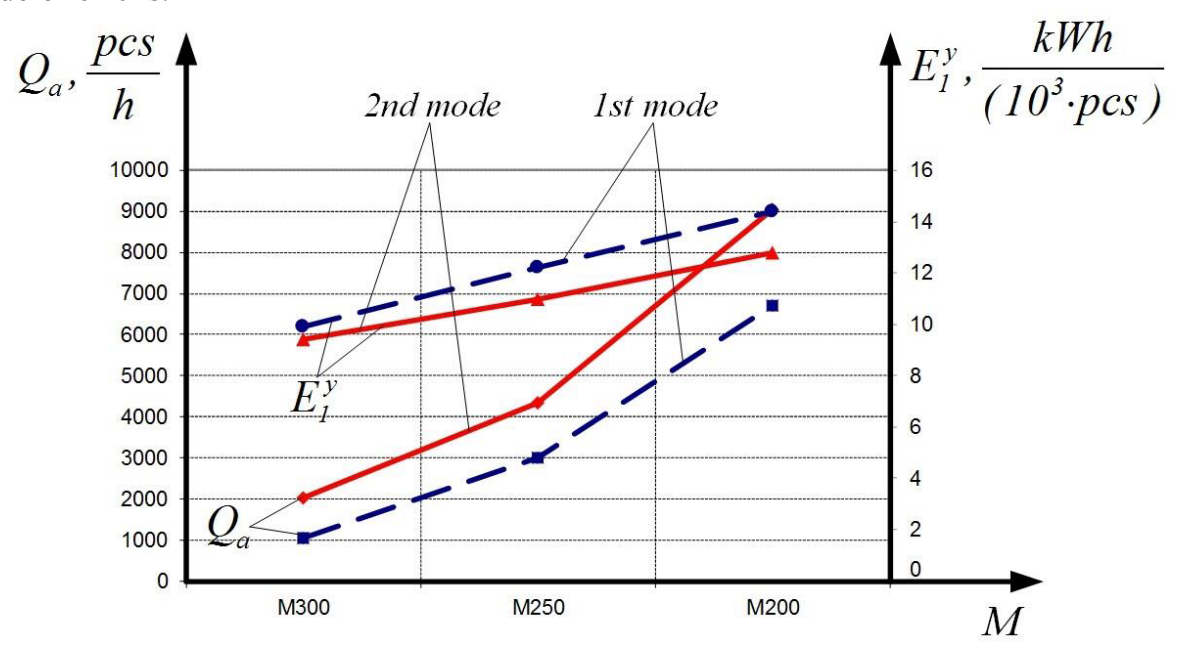

Fig. 3. Comparison of an auger vacuum extruder operation modes.

\section{Conclusions}

The conducted investigation made possible to design MACS EAS (a multidimensional automatic control system of the extruder actuating systems) for shaping ceramic paste while producing bricks with minimum energy consumption and maximum productivity.

The authors also developed a mathematical model of energy processes observed in an auger vacuum extruder when shaping ceramic paste.

The authors assessed the effectiveness of shaping and proved that $K_{Q i}$ and $K_{E i}$ coefficients depend on grade $M_{i}$ of bricks and can rise to $35-93 \%$ и and $4.6-11.2 \%$ respectively.

\section{References}

1. GOST R 55646-2013 Resource Saving. Production of Ceramic Bricks and Stones. Guidance on Implementing of Best Available Techniques for Improving Energy Efficiency and Environmental Performance (Standartinform, Moscow, 2014)

2. S.Ya. Galitskov, M.A. Nazarov, and K.S. Galitskov, International Journal of Applied Engineering, 10(20), 40846-40852 (2015)

3. M.A. Nazarov, V.I. Kichigin, and A.S. Fadeev, Procedia Engineering 153, 509-512 (2016)

4. S.Ya. Galitskov, K.S. Galitskov, and M.A. Nazarov, Industrial and Civil Engineering 3, 25-29 (2014)

5. S.Ya. Galitskov, and M.A. Nazarov, Fundamental Research 8-1, 29-32 (2013)

6. S.Ya. Galitskov, M.A. Nazarov, K.S. Galitskov, and A.P. Maslyanitsyn, Science Review 12, 200-203 (2013)

7. S.Ya. Galitskov, K.S. Galitskov, and M.A. Nazarov, Industrial and Civil Engineering 9, 27-30 (2015)

8. S.Ya. Galitskov, K.S. Galitskov, and M.A. Nazarov, Science Review 14, 213-218 (2015) 
9. K.S. Galitskov, Industrial and Civil Engineering 6, 59-63 (2015)

10. K.S. Galitskov, and M.A. Nazarov, Complex systems: control and modeling problems, 351-355 (2016) 